\title{
Caracterización de tres índices de cosecha de cacao de los clones CCN51, ICS60 e ICS 95, en la montaña santandereana, Colombia
}

\section{Characterization of three indexes of cocoa harvest of the clones CCN51, ICS60 and ICS 95 in the santandereana mountain, Colombia}

\author{
Caracterização dos três índices de colheita de cacau \\ do clones CCN51, ICS60 e ICS 95 na montanha \\ Santander, Colômbia
}

\author{
Lucas Fernando Quintana Fuentes', Salomón Gómez Castelblanco², \\ Alberto García Jerez ${ }^{3} \&$ Nubia Martínez Guerrero ${ }^{4}$ \\ 'ingeniero de Alimentos, Especialista en Dirección de Empresas, Magister en Ingeniería en Sistemas \\ de Calidad y Productividad. ${ }^{2}$ ingeniero de Alimentos, Especialista en Control y Calidad de Alimentos. \\ ${ }^{3}$ Biólogo, Especialista en química ambiental, Magister en Desarrollo sostenible y medio ambiente. \\ ${ }^{4}$ Ingeniera Agrónoma. Magister en Ciencias Agrarias. \\ 1,2,3 Grupo de Investigación GIA. Programa de Ingeniería de Alimentos. \\ Escuela de Ciencias Básicas, Tecnología e Ingenierías. Universidad Nacional Abierta \\ y a distancia UNAD. Carrera 27\#40-43 Bucaramanga. Colombia. \\ 1'ucas.quintana@unad.edu.co, ${ }^{2}$ salomon.gomez@unad.edu.co, \\ 3alberto.garcia@unad.edu.co, ${ }^{4}$ nubiamartinezg@yahoo.es
}

\section{Resumen}

En Colombia la región con mayor producción de cacao es la denominada montaña santandereana, que se ubica entre los pisos térmicos cálido y templado donde se cultiva cacao entre los 200 a 1200 msnm. en los departamentos de Santander y Norte de Santander. Esta investigación surge como una necesidad de la Federación nacional de cacaoteros, Fedecacao, con el propósito de construir herramientas que permitan a futuro tomar decisiones sobre el manejo poscosecha del fruto, buscando una mejora continua de la calidad e incrementando así la competitividad en los mercados. El objetivo fue evaluar tres variables: índice de mazorca, índice de grano y porcentaje de cascarilla, según la Norma Técnica Colombiana 1252 , en tres franjas altitudinales de la montaña san- tandereana, para tres clones introducidos: CCN 51, ICS 60 e ICS 95. El seguimiento y análisis de las tres variables se registró en dos cosechas denominadas principal e intermedia, en tres fincas para cada franja altitudinal y en los tres clones. Se determinó la manera cómo esta condición influye en el resultado de estas variables relacionadas con el rendimiento y la calidad, mediante la evaluación de los datos obtenidos a través de métodos estadísticos de correlación y de determinación, estableciéndose que no hay diferencias significativas que permitan concluir que la altitud influye en estas variables de calidad.

Palabras clave: cacao, índices, clon, calidad, franja altitudinal. 


\section{Abstract}

In Colombia the region with the greatest production of cocoa is the so-called santandereana mountain, which is located between the thermal floors warm and temperate where cocoa is cultivated between 200 to $1200 \mathrm{msnm}$ in the departments of Santander and Norte de Santander. This research arises as a need for the national federation of cocoa, Fedecacao, with the purpose to build tools that enable future take decisions on the postharvest handling of the cocoa, looking for a continuous improvement of the quality and also increase competitiveness in the markets. The objective was to evaluate three variables: index of cob, index of grain and percentage of husk, according to the Colombian Technical Standard 1252, into three altitudinal bands of the mountain Santandereana, for three clones introduced: CCN 51, ICS 60 and ICS 95. The monitoring and analysis of the three variables was recorded in two harvests called main and intermediate, on three farms for each altitudinal band and in the three clones. It was determined how this condition affects the outcome of these variables related to the performance and quality, by evaluating the data obtained through statistical methods of correlation and determination, with the stipulation that there are no significant differences that could lead to the conclusion that the altitude has an impact on these quality variables.

Keywords: cocoa, indexes, clone, quality, altitudinal band

\section{Resumo}

$\mathrm{Na}$ Colômbia, a região com a maior produção de cacau é chamado, montanha santander, que fica entre as zonas climáticas quentes e temperadas, onde o cacau é cultivado entre 200 a 1200 metros acima do nível do mar, nos departamentos de Santander e Norte Santander. Esta pesquisa vem como uma necessidade da Federação Nacional de cacau, Fedecacao, a fim de construir ferramentas para permitir futuras decisões sobre o manuseio pós-colheita dos frutos, à procura de uma melhoria contínua da qualidade e aumentando a competitividade do mercado . O objetivo foi avaliar três variáveis: índice de vagem, índice e percentuais de casca. De acordo com a Norma Técnica Colombiana 1252, em três faixas de altitude das montanhas Santander, três clones introduzidos: CCN 51, ICS 60 e ICS 95. A monitorização e análise das três variáveis foi gravada em duas colheitas chamadas primária e intermediária, em três fazendas para cada faixa de altitude e os três clones. Determinou-se como esta condição afeta o resultado dessas variáveis relacionadas ao desempenho e qualidade através da avaliação de dados obtidos através de métodos estatísticos de correlação e determinação, estabelecendo que não existem diferenças significativas para concluir que o altitude afeta estas variaveis de qualidade.

Palavras-chave: cacau, índices, clone, qualidade, faixa altitudinal

\section{Introducción}

En Colombia la región con mayor producción de cacao es la denominada montaña santandereana, la cual se ubica entre los pisos térmicos cálido y templado, altura óptima en donde se cultiva el cacao entre los 200 a 1200 msnm, esta región está comprendida por los departamentos de Santander y Norte de Santander (Fedecacao, 2012).
Esta investigación surge como una necesidad del gremio de cacaoteros que asociados en la Federación nacional de cacaoteros, Fedecacao, y con el propósito de construir herramientas que permitan a futuro tomar decisiones sobre el manejo poscosecha, buscando una mejora continua de la calidad del fruto e incrementando así la competitividad del fruto en los mercados. 
La zona objeto de estudio, está ubicada en el municipio de San Vicente de Chucuri, departamento de Santander, Colombia. Este departamento se considera el mayor productor de cacao del país, ya que contribuye con un 37\% de la producción nacional, también cuenta esta zona con la posibilidad de tener producción en una franja altitudinal que va desde los 200 msnm a los $1300 \mathrm{msnm}$. La economía agropecuaria chucureña se ha basado históricamente en el sistema productivo del cacao y en menor proporción del café. El cultivo del cacao en esta región corresponde a un tipo de explotación de economía campesina, se desarrolla sobre todo en fincas de mediana y pequeña propiedad. El nivel tecnológico de este cultivo oscila entre medio a bajo. El sistema de producción se caracteriza por la interacción de varias especies dentro del mismo arreglo tales como aguacate, cítricos y plátano. El sistema de siembra con sombrío es una práctica de conservación que permite aprovechar mejor los suelos y el agua. Adicionalmente, los clones de cacao objeto de esta investigación se encuentran presentes en todas las veredas del municipio de San Vicente de Chucuri, y han sido recomendados por entidades como Fedecacao por sus características productivas, se tiene que el clon CCN 51 cuenta con un $25 \%$ de participación, el ICS 60 con un $14 \%$ y el ICS 95 con un $25 \%$, de la producción del departamento, como se muestra en la Figura 1 (Rincón, 2007; Corpoica, 2000).

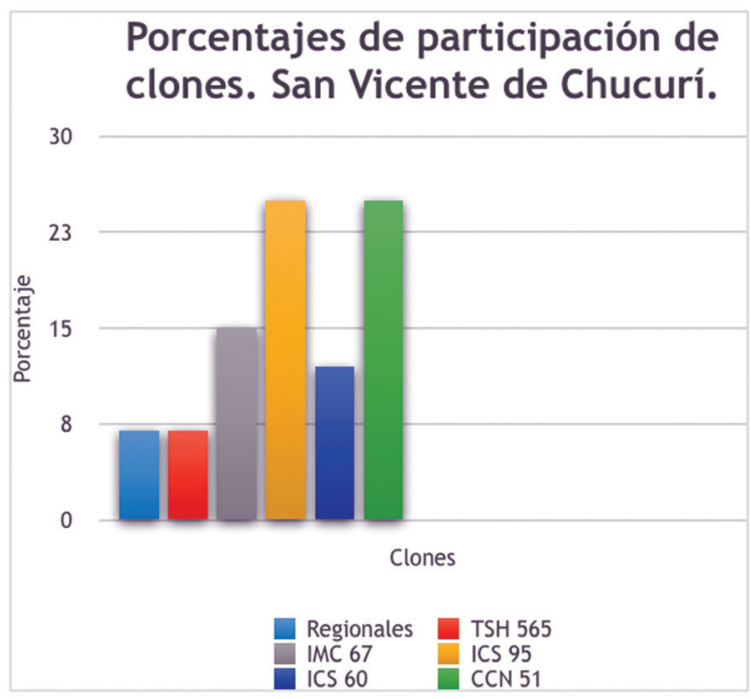

Figura 1. Producción de los clones de cacao del municipio de San Vicente de Chucurí.

Fuente: Censo cacaotero 2007.

En las zonas productoras de cacao el nivel de productividad se mide por el rendimiento agronómico, siendo el ideal $1500 \mathrm{~kg} \mathrm{ha}^{-1}$, valor con el cual se logra el punto de equilibrio y se hace rentable la producción; en Colombia esta producción se encuentra alrededor de los $450 \mathrm{~kg} \mathrm{ha}^{-1}$, resultando muy poco productivo a pesar de tener una alta superficie plantada de 100.000 ha, como principales causas de esta baja producción se consideran el bajo número de árboles por ha, la baja fertilidad del material genético y la edad avanzada de los cultivos. (Industria y comercio superintendencia, 2012)
La importancia de los clones seleccionado, en cuanto a productividad, resistencia a enfermedades y calidad sensorial es algo a resaltar y al evaluar los índices de mazorca, índice de grano y porcentaje de cascarilla se potencia su aprovechamiento en todo el país, teniendo en cuenta que el cacao es un cultivo que beneficia principalmente a comunidades campesinas y a pequeños productores. (Montoya et al., 2015)

Adicionalmente a esto se tiene que la comercialización del grano para plantas productoras de 
licor de cacao o chocolate se ve afectada por los rendimientos que puede dar el grano en cuanto a su tamaño o índice de grano y a su porcentaje de cascarilla, índices que una vez procesados los granos afectan los rendimientos al interior de la planta procesadora. (Baena \& García, 2012)

La cascarilla es un alto porcentaje (11-14\%) de residuo en las plantas procesadoras de cacao tanto en Colombia como a nivel mundial. Actualmente han aumentado estudios relacionados para este tipo de residuos y su posible utilización, debido a que éstos representan un importante componente de los residuos agrícolas y desechos agroindustriales en el mundo, constituyendo una buena fuente de recursos renovables y energía. (Baena \& García, 2012; Ayeni, 2010; Becket, 1998).

Existen tipos de cacao criollo, forastero e híbridos.
El criollo: es el más fino, caracterizado por su agradable sabor y exquisita aroma, susceptible a plagas y enfermedades. El tipo forastero: posee un buen rendimiento en contenido de grasa, es de apariencia amelonada, el sabor es amargo y poco consistente. Los tipos híbridos: son cruce sexual de dos árboles y los clones, pertenecen a un material genético uniforme, derivado de un individuo y propagado solo por medios vegetativos.

El concepto de clon no significa que todas las plantas de un mismo clon, sean idénticas fenotípicamente en todas sus características. El comportamiento de una planta depende de la interacción genotipo-ambiente. En consecuencia, una planta puede varíar la apariencia, la producción, los frutos o almendras de acuerdo con el clima, suelo, agua, enfermedades u otras causas. Los clones más conocidos se describen en la Tabla1.

Tabla 1. Nomenclatura, nombres y origen de los clones de cacao.

\begin{tabular}{llccc}
\hline \multicolumn{1}{c}{ CLON } & \multicolumn{1}{c}{ NOMBRE } & TIPO GENETICO & AO & ORIGEN \\
\hline ICS & IMPERIAL COLLEGE SELECTION & HIBRIDO TRINITARIO & 1931 & TRINIDAD \\
IMC & IQUITOS MARAÑON COLLECTION & AMAZONICO & 1938 & PERÚ \\
\hline UF & UNITED FRUIT CO. & HIBRIDO TRINITARIO & -- & TRINIDAD \\
TSH & TRINIDAD SELECTION HYBRID & SCA 6 X IMC 67 & 1957 & TRINIDAD \\
TSA & TRINIDAD SELECTION AMAZON & SCA 6 X IMC 67 & 1957 & TRINIDAD \\
CAP & CENTRO AGROPECUARIO PICHILINGUE & ICS 1 X ICS 6 & 1955 & TRINIDAD \\
CCN & COLECCIÓN CASTRO NARANJAL & ICS 95 X IMC 67 & 1965 & ECUADOR \\
EET & ESTACION EXPERIMENTAL TROPICAL & FAL X DESCONOCIDO & 1945 & ECUADOR \\
PA & PARINARI & AMAZONICO & 1930 & PERÚ \\
P & POUND & AMAZONICO & 1943 & PERÚ \\
SCA & SCAVINA & AMAZONICO & 1930 & PERÚ \\
PORCELANA & PORCELANA & CRIOLLO & & VENEZUELA \\
\hline
\end{tabular}

Fuente: Fedecacao (2012) 
Algunos autores han descrito cambios en los índices de cosecha con la franja altitudinal en donde es cultivado el grano, sin embargo, en el departamento de Santander no se cuenta con referentes de este tipo por lo cual se decidió realizar la presente investigación. El objetivo fue evaluar el comportamiento de tres variables relacionadas con la producción y características físicas del grano de cacao, según la Norma Técnica Colombiana 1252 (Tabla 2), como son el índice de mazorca, el índice de grano y el porcentaje de cascarilla en tres franjas altitudinales de la montaña santandereana, para tres clones introducidos: CCN 51, ICS 60 e ICS 95.

Tabla 2. Requisitos del grano de cacao, de acuerdo a la norma técnica colombiana, NTC 1252.

\section{Requisitos}

Contenido de humedad en\%(m/m)

Contenidos de impurezas o meterías extrañas en \%(m/m)

Grano mohoso interno, numero de granos /100 gramos máx.

Granos dañados por insectos y/o germinados, numero de granos /100 gramos máx.

Contenido de pasilla, numero de granos /100 gramos máx.

Contenido de almendra \%(m/m), min.

Masa (peso), en g/100 gramos, min.

Granos bien fermentados, numero de granos /100 gramos min.

Granos insuficientemente fermentados, numero de granos /100 gramos máx.

Granos pizarrosos, numero de granos /100 gramos máx.

Fuente: Norma Técnica Colombiana, NTC 1252.

\section{Materiales y métodos}

El modelo estadístico adoptado en el proceso fue al azar, realizado en tres franjas altitudinales la primera comprendida entre los 200-500 msnm, la segunda entre los 501-800 msnm y la tercera entre los $801-1200 \mathrm{msnm}$, con tres repeticiones en 3 fincas por cada franja altitudinal. Para un total de 9 unidades experimentales, en dos cosechas principal e intermedia, evaluando las variables siguientes el índice de mazorca, índice de grano y porcentaje de cascarilla. Los genotipos seleccionados CCN 51, ICS 60 e ICS 95 son los materiales de mayor presencia en los diferentes pisos altitudinales del municipio de San Vicente de Chucuri.

\section{Análisis físico de las muestras}

De acuerdo a lo establecido en la Norma Técnica Colombiana NTC 1252 se realizaron los siguientes análisis:

\section{Índice de mazorca}

Se procede a pesar el total de la muestra seca, y teniendo en cuenta que cada muestra se realizó con 20 mazorcas, se halla el número de mazorcas que se necesitan para obtener un $\mathrm{kg}$ de cacao seco para cada uno de los clones (Fedecacao, 2004; Stevenson \& Corven, 1993) 
Índice de grano

Se tomaron cuatro muestras de 100 granos escogidos al azar, se pesó cada sub-muestra y se calculó un promedio de las cuatro, este promedio se dividió en 100 y el valor fue el índice de grano de cada una de os clones (Icontec, 2003; Stevenson \& Corven, 1993).

\section{Porcentaje de cascarilla}

De cada muestra se tomaron y pesaron 50 granos, a los cuales se les retiro la cascarilla y se colocaron en recipientes separados cascarilla y cotiledón, posteriormente se pesaron cascarilla y cotiledón, con estos datos se halló el porcentaje de cascarilla teniendo en cuenta el dato del peso inicial de los 50 granos (Fedecacao, 2004; Ica, 2003; Stevenson \& Corven, 1993).

\section{Selección de Fincas y muestreo}

Se identificaron y geo-referenciaron las fincas de cada franja altitudinal donde se encontraron los tres materiales propuestos (Figura 1). Los materiales fueron transportados hasta la Granja Villa Mónica de propiedad de Fedecacao ubicada en el municipio de San Vicente de Chucuri - Santander, donde se realizó el proceso de Poscosecha bajo un mismo protocolo establecido por Fedecacao para todas las muestras, que consistió en la cosecha de sólo mazorcas maduras y sin enfermedades, partido con herramienta adecuada para no causar daño a los granos de cacao y desgranado en recipientes no metálicos y limpios, fermentado en cajón de madera por 144 horas con un primer volteo a las 48 horas y dos volteos seguidos cada 24 horas, una vez fermentados se procede al secado en elba hasta lograr un porcentaje final de humedad del 7\% (Fedecacao, 2012)

En el mapa presentado en la Figura 2 se señalan las veredas en donde fueron tomadas las muestras, y en la Tabla 3 se relacionan las fincas correspondientes a cada franja altitudinal en la zona de estudio del municipio de San Vicente de Chucuri.

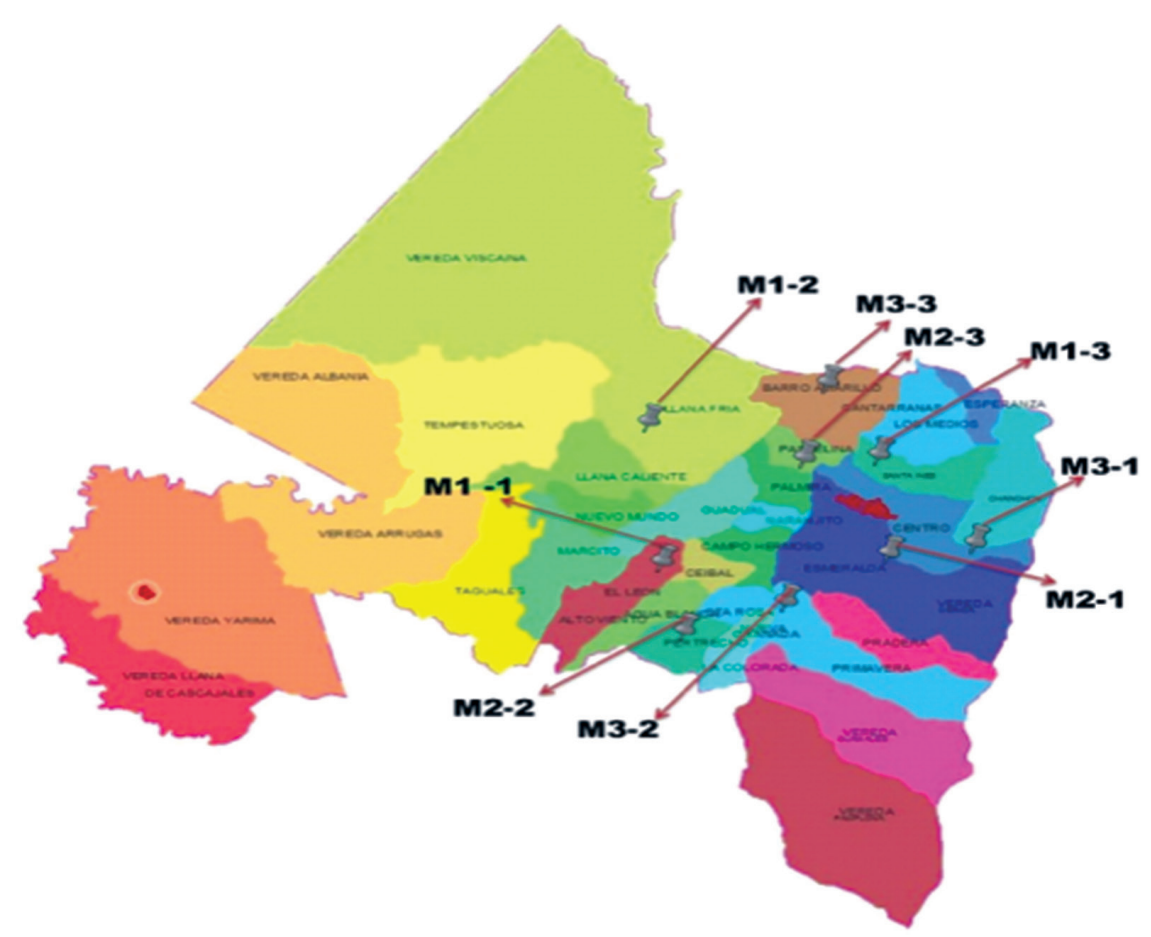

Figura 2. Mapa de zonas de muestreo en el municipio de San Vicente de Chucuri. Santander, Colombia. 
Tabla 3. Relación fincas Vs franja altitudinal de la zona de estudio.

\begin{tabular}{ll}
\hline Franja altitudinal & \multicolumn{1}{c}{ Fincas } \\
\hline $200-500 \mathrm{msnm}$ & $\begin{array}{l}\text { Montecarmelo - } \\
\text { Maranduda - San Luis }\end{array}$ \\
$501-800 \mathrm{msnm}$ & $\begin{array}{l}\text { Villa Mónica - tres aljibes } \\
\text { - San Jorge }\end{array}$ \\
$801-1200 \mathrm{msnm}$ & $\begin{array}{l}\text { Altamira - Macondo - } \\
\text { El Paraíso }\end{array}$ \\
\hline
\end{tabular}

\section{Fermentación y Secado}

Se cosecharon por cada muestra 20 mazorcas; para el proceso de fermentación, se colocaron los granos de cacao en bolsas plásticas especiales con orificios, que permitieron el drenado de los jugos y el contacto de la muestra con la masa total de fermentación. El manejo de la fermentación se hizo siguiendo las indicaciones de Fedecacao (2004, 2012), que recomienda la primera remoción de la masa a las 48 horas y posteriormente remociones cada 24 horas hasta completar entre $140 \mathrm{y}$ 144 horas de fermentación, en total 4 volteos a las $48,72,96$ y 120 horas.

El proceso de secado se realizó sobre una estructura de madera, conocida en la región con el nombre de elba; para el primer día de secado las muestras se expusieron al sol por dos horas, haciendo remociones de la masa cada 20 minutos, al segundo día, durante cuatro horas, haciendo remociones cada 20 minutos, para el tercer día durante todo el día, es decir más o menos 8 horas, hasta llegar a una humedad del $7 \%$.

\section{Resultados}

\section{Índice de mazorca}

Para este índice y de acuerdo a los resultados mostrados en la Tabla 4, se observa que no hay una correlación entre las franjas altitudinales y el índice de mazorca, a excepción del ICS 95 con una correlación negativa, pero al determinar el coeficiente de determinación da una influencia del $31 \%$ con lo cual se deduce que no hay relación con la altitud, y que los índices están en los rangos establecidos por Perea et al. (2013) en su trabajo de caracterización de cultivares para estos tres clones de cacao.

En la segunda cosecha se mantiene que no hay correlación para los clones CCN 51 e ICS 60, pero en cambio se presenta una correlación negativa para el clon ICS 95, con un porcentaje de influencia de la altura en una $51 \%$. Se concluye entonces que no hay una fuerte influencia de la altura en este índice.

Tabla 4. Índice de correlación para el índice de mazorca.

\begin{tabular}{|c|c|c|c|c|c|c|}
\hline \multicolumn{7}{|c|}{ INDICE DE MAZORCA } \\
\hline & \multicolumn{3}{|c|}{ PRIMERA COSECHA } & \multicolumn{3}{|c|}{ SEGUNDA COSECHA } \\
\hline & CCN 51 & ICS 60 & ICS95 & CCN51 & ICS60 & ICS95 \\
\hline r & 0,18 & $-0,3$ & $-0,56$ & 0,05 & 0,03 & $-0,72$ \\
\hline$r^{2}$ & 0,03 & 0,09 & 0,31 & 0 & 0 & 0,51 \\
\hline
\end{tabular}

Se observa en la Figura 3 que el índice de mazorca más alto lo tiene el ICS 95 debido a su tamaño de grano, Perea et al., 2013 establecieron un promedio cercano a 20 mazorcas por kg para este clon, se obtuvo un rango para este índice de 19 a 28 mazorcas para obtener un $\mathrm{kg}$ de grano seco. 
Para el CCN 51 se ha considerado un promedio de 15 mazorcas y en esta investigación se obtuvo un intervalo 14 a 17, a su vez para el ICS 60 un

A

INDICE DE MAZORCA PRIMERA COSECHA

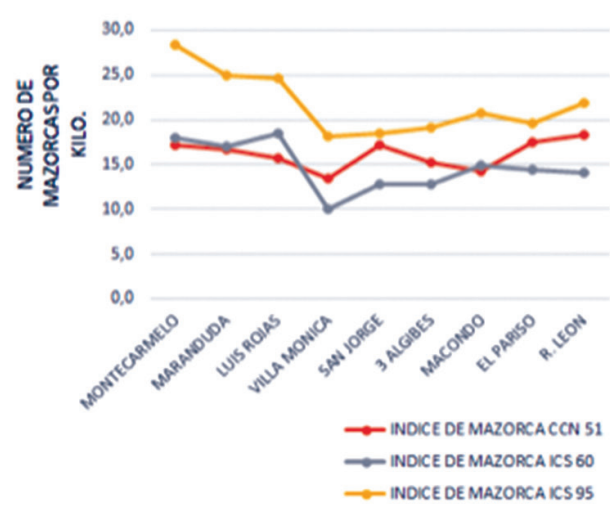

promedio de 13 mazorcas por su tamaño de grano y se presentó un intervalo 12 a 17 mazorcas por kg de grano seco.

B

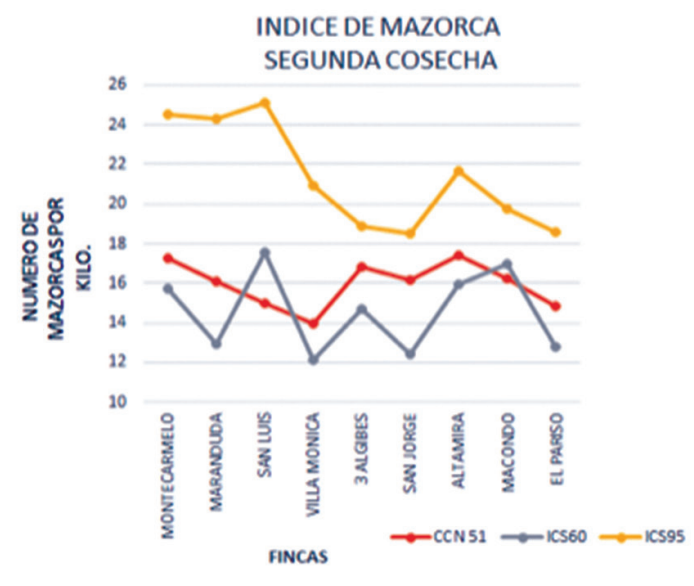

Figura 3. Comportamiento del índice de mazorca para los tres clones de cacao.

\section{Consolidado de las dos cosechas}

Al observar la ecuación de la recta en la Figura 4 se determina que hay una relación directa pero con muy baja correlación y con un muy bajo porcentaje de variación de acuerdo al modelo de $2 \%$ en la Figura 4 A.
Para el clon ICS60 hay una relación inversa con muy bajo coeficiente de correlación y con un muy bajo porcentaje de variación del 3.16\% (Figura 4 B). Al igual que el anterior, el clon ICS95 presenta una relación inversa, con un porcentaje de variación e acuerdo al modelo determinado del $42 \%$ (Figura $4 \mathrm{C}$ ).
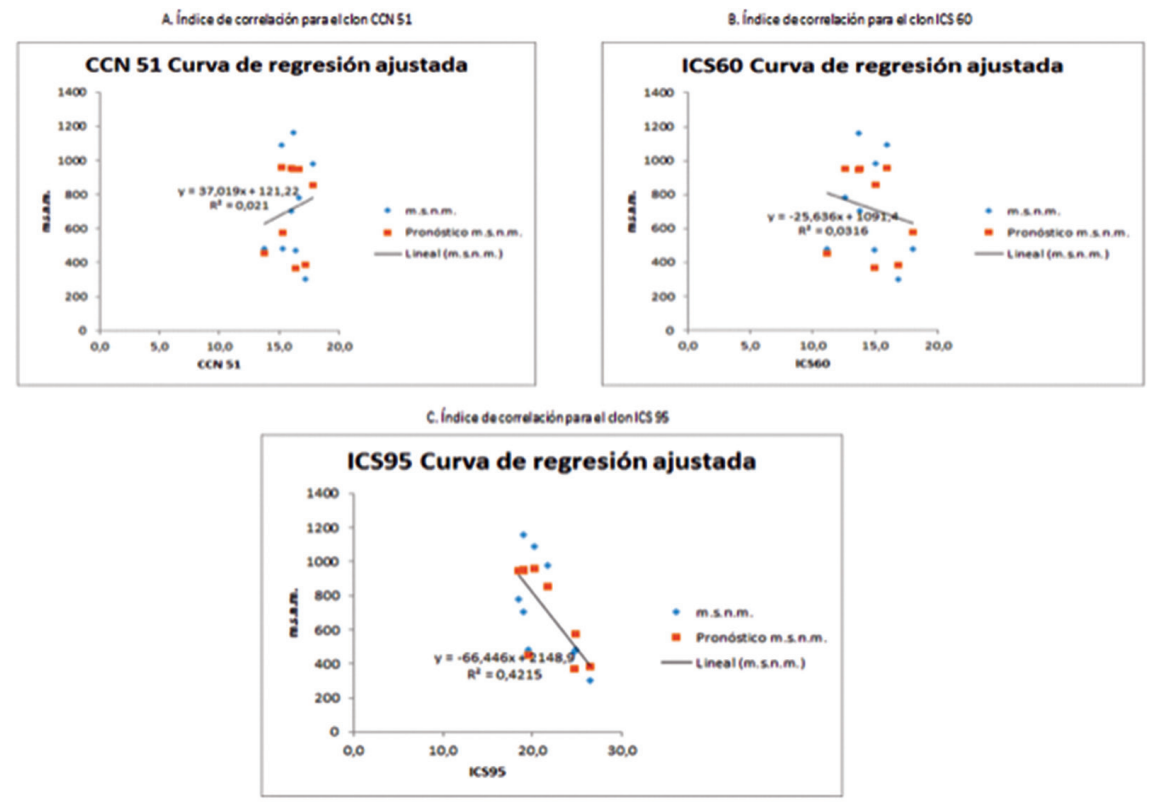

Figura 4. Índice de correlación correspondiente al índice de mazorca, para cada clon. 


\section{Índice de grano}

En la primera Cosecha en este indicador de calidad física se observa un comportamiento lineal sin influencia marcada por la variable de la altitud (Tabla 5), el máximo porcentaje de influencia es de $29 \%$ para el ICS 95 , lo que indica que la altitud no influye significativamente en este ín- dice, por lo tanto en esta primera cosecha no hay correlación entre las franjas altitudinales y el índice de grano.

En la segunda cosecha no se observa correlación marcada, por lo tanto se concluye que no hay influencia de la altitud sobre el tamaño del grano para ninguno de los tres clones.

Tabla 5. Índice de correlación para el índice de grano.

\begin{tabular}{|c|c|c|c|c|c|c|}
\hline \multicolumn{7}{|c|}{ INDICE DE GRANO } \\
\hline & \multicolumn{3}{|c|}{ PRIMERA COSECHA } & \multicolumn{3}{|c|}{ SEGUNDA COSECHA } \\
\hline & CCN 51 & ICS 60 & ICS95 & CCN51 & ICS60 & ICS95 \\
\hline r & $-0,14$ & $-0,34$ & 0,54 & 0,04 & 0,43 & 0,28 \\
\hline$r^{2}$ & 0,02 & 0,12 & 0,29 & 0,16 & 0,18 & 0,08 \\
\hline
\end{tabular}

Al revisar la Figura 5 se observa que tanto para la Figura $5 \mathrm{~A}$ y la $5 \mathrm{~B}$, no hay una tendencia marcada a subir o a bajar con la altitud, se mantienen adicionalmente los resultados dentro de lo establecido para cada clon, en el caso del CCN 51 se ha determinado un índice de grano promedio de 1,6 g de acuerdo a Perea et al., 2013 encontrándose un rango 1.2 a $1,7 \mathrm{~g}$, para el ICS 60 un grano grande con promedio de $2,3 \mathrm{~g}$ y con un rango de 1,5 a 2,4 g y por ultimo para el ICS 95, clon considerado como grano pequeño, un índice promedio de $1,4 \mathrm{~g}$ y con un rango de 1.1 a $1,5 \mathrm{~g}$.
A

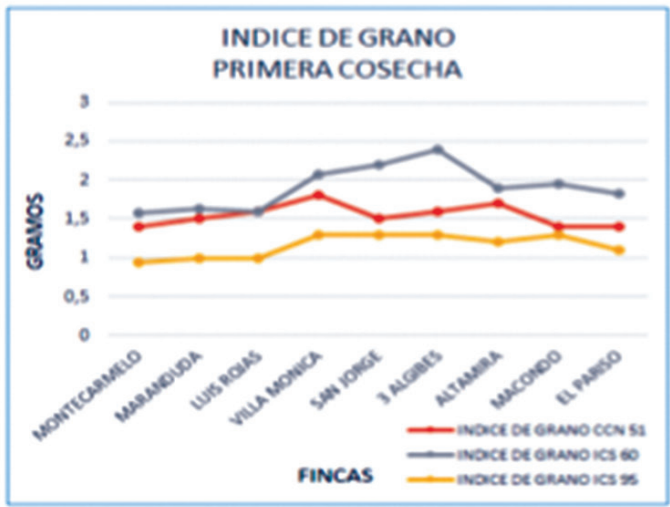

B

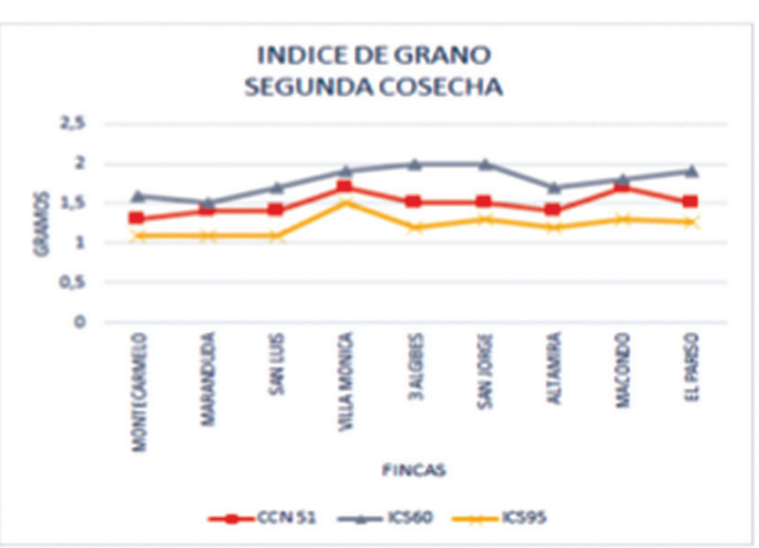

Figura 5. Comportamiento del índice de grano para los tres clones de cacao. 
Se puede observar en la gráfica 6 una relación positiva pero de poca significancia con un porcentaje de variación del $1.14 \%$ como se observa en el Figura 6 A. En la misma Figura 6 A, para el ICS 60 se tiene una relación positiva y con un porcentaje de variación del $15 \%$ que demues- tra una muy baja correlación como se observa en la Figura 6 B. En tanto, hay una relación positiva pero muy baja, lo que indica que no hay correlación significativa y con un porcentaje de variación del $16.69 \%$, correspondiente al ICS 95 (Figura $6 \mathrm{C}$ ).
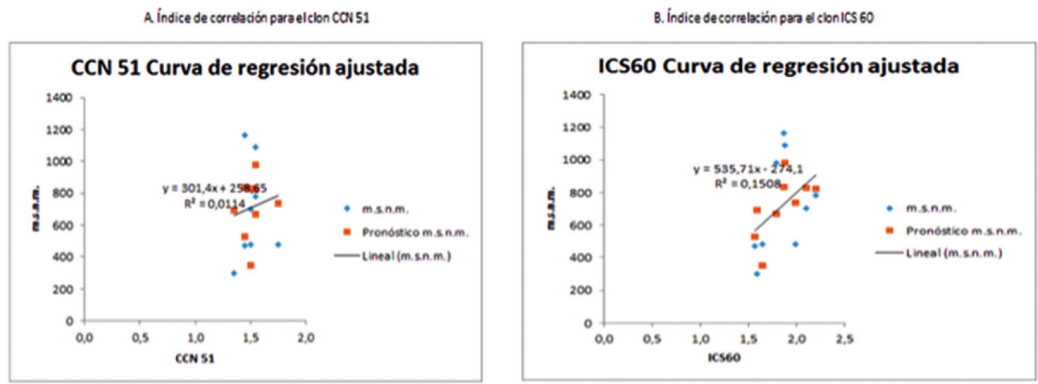

C. índice decorrelixión pan el don les 95

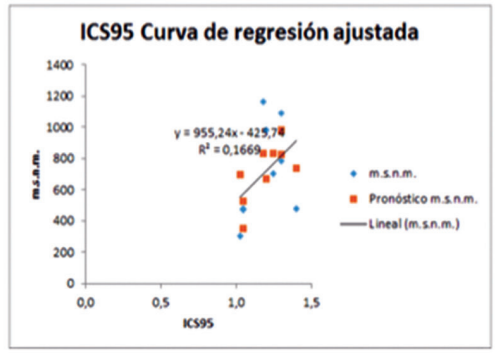

Figura 6. Índice de correlación correspondiente al índice de grano, para cada clon.

\section{Porcentaje de cascarilla}

Como se muestra en la Tabla 6, en la primera cosecha el porcentaje de cascarilla observado se mantiene dentro de los porcentajes revisados en Becket (1998), los cuales están dentro de 10\% a 14\% para granos normales y $16 \%$ para granos pequeños tipo ICS 95 o pasilla; para granos con índice de grano mediano o grande se observa que en este caso es inverso al tamaño del grano, a mayor tamaño menor porcentaje de cascarilla lo cual es de beneficio para el procesador en la producción de licor de cacao. Se observa que el porcentaje de cascarilla no tiene una variación pronunciada con la altitud, se presenta en la segunda cosecha una correlación negativa para el ICS 96 influenciada en un $39 \%$. Se puede concluir que en este caso la altitud no influye y que la variación puede deberse a otras causas.

Tabla 6. Índice de correlación para porcentaje de cascarilla

\begin{tabular}{|c|c|c|c|c|c|c|}
\hline \multicolumn{7}{|c|}{ PORCENTAJE DE CASCARILLA } \\
\hline & \multicolumn{3}{|c|}{ PRIMERA COSECHA } & \multicolumn{3}{|c|}{ SEGUNDA COSECHA } \\
\hline & CCN 51 & ICS 60 & ICS95 & CCN51 & ICS60 & ICS95 \\
\hline$r$ & $-0,39$ & $-0,35$ & $-0,37$ & $-0,44$ & $-0,5$ & $-0,62$ \\
\hline$r^{2}$ & 0,15 & 0,12 & 0,14 & 0,19 & 0,25 & 0,39 \\
\hline
\end{tabular}


Se observa en la Figura 7, un comportamiento con respecto al porcentaje de cascarilla que es inverso al tamaño del grano como esta descrito en el anterior índice, a menor tamaño de grano mayor porcentaje de cascarilla, según la caracterización realizada por Perea et al., 2013 y deZaam, 2009, se tiene que para el ICS 95, el
ICS 60 y el CCN 51 se deben obtener valores promedio entre $11-12 \%$ es decir un porcentaje de cascarilla medio. Se observa que para el ICS 95 hay un rango desde el 13 al $16 \%$, para el ICS 60 del 10 al 14\% y para el CCN 51 del 12 al 14\% siendo este último el más consistente y con menor variación.
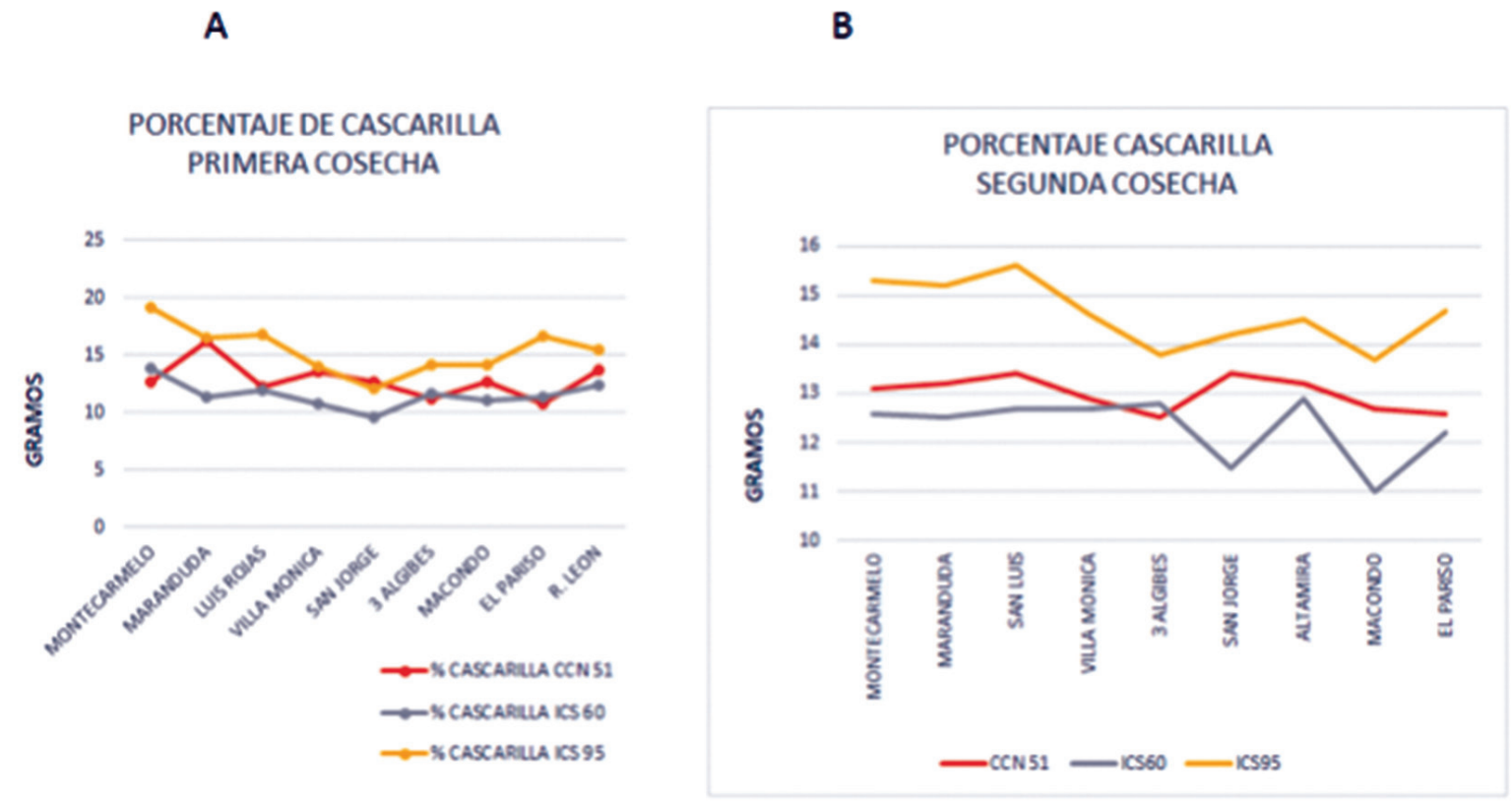

Figura 7. Comportamiento del porcentaje de cascarilla para los tres clones de cacao

La Figura 8 presenta una relación inversa en la Figura $8 \mathrm{~A}$, poco significativa, no hay una relación directa entre la altitud y el porcentaje de cascarilla, con un porcentaje de variación del modelo del $18.03 \%$. Se observa una correlación inversa con un nivel de correlación muy bajo en la Figura 8 B que permite establecer que no hay relación significativa y adicionalmente con un porcentaje de variación muy bajo del $21.32 \%$.

La correlación es inversa para el clon en la Figura $8 \mathrm{C}$ que arroja una muy bajo porcentaje de variación del $18.44 \%$, por lo cual no hay una relación del porcentaje de cascarilla de acuerdo a la altitud. 
A indize de correlodión parsel clon CoV 51

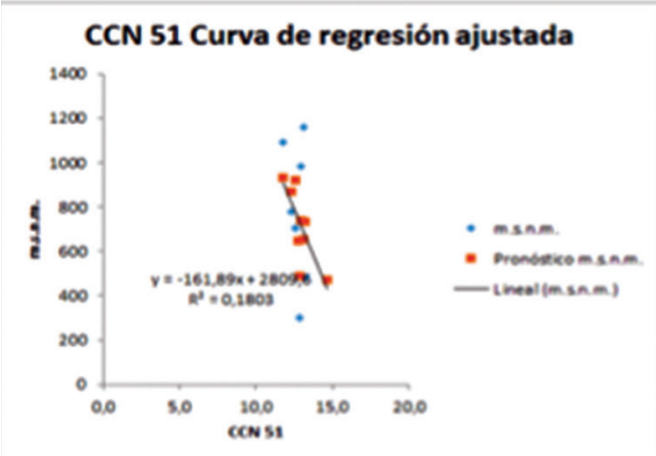

B. indice decorrebción pars el clen|CS 60

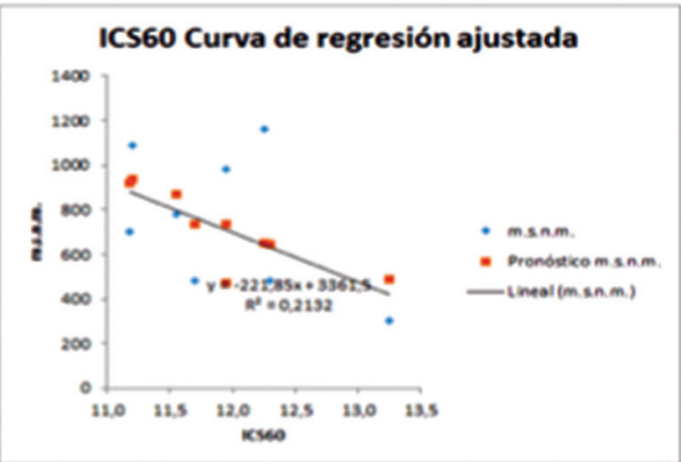

C indice de coerdxiónpanel denicss

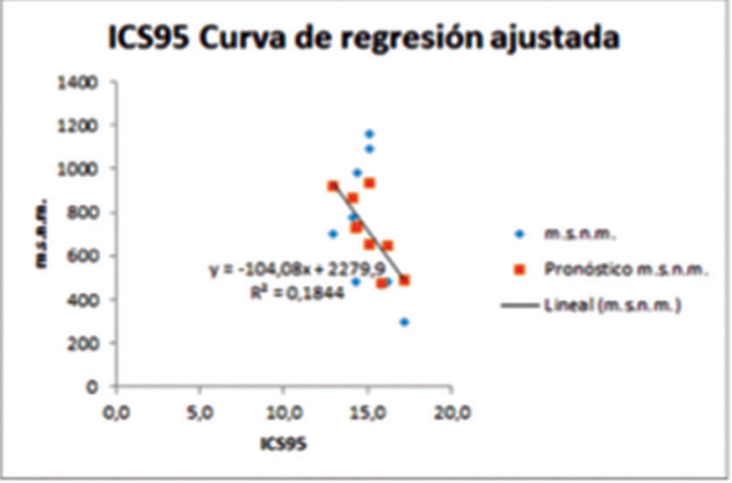

Figura 8. Índice de correlación correspondiente al porcentaje de cascarilla, para cada clon.

\section{Conclusiones}

Los clones son parte de la estrategia genética para mejora de los cultivos de cacao, que permite que se adapten a diferentes condiciones ambientales dando como resultado variación en los fenotipos, el fruto y en las características físicas, químicas y sensoriales, que influirán directamente en que este cultivo sea productivo y genere los rendimientos esperados.

Se puede establecer con los resultados obtenidos que para razones de productividad teniendo como referente el índice de mazorca, el clon más productivo sería el ICS 60 , siguiendo en su orden el clon CCN 51 con índices aceptables, comprendidos entre 16 y 20 mazorcas por $\mathrm{kg}$ como lo establece Perea et al. (2013) y como último el clon ICS 95 con un índice aceptable para el cual se necesita un número mayor a las 21 mazorcas para producir un $\mathrm{kg}$ de cacao seco.
El índice de grano como segundo factor de productividad estudiado es referente al logro en planta de mayores rendimientos muy importante para los procesos de tostado y molido como lo establece Becket (1993) y para los clones de estudio se puede concluir que son notorias las diferencias, se encuentra al ICS 60 con un índice de grano alto superior a los $1,7 \mathrm{~g}$, el CCN 51 como un clon de índice de grano medio entre 1,4 y $1,6 \mathrm{~g}$, y el clon ICS 95 con un índice de grano bajo inferior a los $1,3 \mathrm{~g}$, esto teniendo en cuenta la clasificación establecida en la caracterización de clones realizada por Perea et al., (2013) y que coincide con los resultados obtenidos.

El porcentaje de cascarilla es un índice de vital importancia para el cálculo de rendimiento en planta, se ha clasificado dentro de los clones con alto contenido de cascarilla al clon ICS 95 con valores superiores al 13\%, a los clones ICS 60 y CCN 51 
con valores intermedios entre los $11-12 \%$, y efectivamente en los resultados se observa la consistencia con esta clasificación manteniéndose en su orden de mayor porcentaje de cascarilla el ICS 95, ICS 60 y el CCN 51.

En términos generales se puede concluir que en esta investigación se determinó que no hay una influencia significativa en el comportamiento de los tres índices estudiados y que en las condiciones del mismo, el cacao obtenido por clon es consistente en la franja altitudinal de los 200 a $1200 \mathrm{msnm}$.

\section{Literatura citada}

1. Ayeni, L.S. (2010). Effect of Combined Cocoa Pod Ash and NPK Fertilizer on Soil Properties, Nutrient Uptake and Yield of Maize (Zea mays). Journal of American Science, 6(3), 79-84.

2. Baena, L. \& García, N. (2012). Obtención y caracterización de fibra dietaría a partir de cascarilla de las semillas tostadas de Theobroma cacao L. De una industria chocolatera Colombiana (Tesis de pregrado) Químico industrial, Universidad Tecnológica de Pereira.

3. Beckett, S.T. (1998). Fabricación y utilización Industrial del chocolate. Editorial Acribia.

4. Corpoica (2000). Caracterización y tipificación de los productores de Cacao del Departamento de Santander. Bucaramanga. Corpoica.
5. deZaan (2009). Cocoa \& Chocolate Manual. 40TH Anniveversary edition. Suiza: ADM Cocoa.

6. FEDECACAO. (2004). El beneficio y características físico químicas del cacao. Bogotá D.C. Produmedios.

7. FEDECACAO. (2012). Guía técnica para el cultivo del cacao. (Quinta Edición ed.). Bogotá Colombia. Ediciones LCB Ltda.

8. ICONTEC (2003). Norma Técnica Colombiana, NTC 1252. Cacao en Grano. Bogotá D.C. ICONTEC.

9. Industria y Comercio Superintendencia. (2011). Cadena productiva del cacao: diagnóstico de libre competencia. Recuperado de: http://www.sic.gov.co/drupal/sites/default/files/files/Cacao.pdf.

10. Montoya, I., Montoya, L. \& Lowy, P. Oportunidades para la actividad cacaotera en el municipio de Tumaco, Nariño, Colombia. Entramado. Enero - Junio, 2015 vol. 11, no. 1, p. 48-59. Recuperado de:http://dx.doi. org/10.18041/entramado.2015v11n1.21107

11. Perea, A., Martínez, N., Aránzazu, F. \& Cadena, T. (2013). Características de calidad del cacao e Colombia. Catálogo de 26 cultivares. (Primera Edición ed.). Bucaramanga - Colombia: División de publicaciones UIS.

12. Rincón, Diego (2007). Censo cacaotero de Santander. Bucaramanga. Fedecacao.

13. Sampieri, R., Fernández-Collado, C. \& Baptista, L. (2006). Metodología de la Investigación. México, D.F. McGraw-Hill Interamericana.

14. Stevenson, C. \& Corven, J. (1993). Manual para el análisis de cacao en el laboratorio. San José, Costa Rica: IICA.

\section{Conflicto de Intereses}

Los autores declaran no tener ningún conflicto de intereses 
